\title{
PROPERTIES OF ELLIPTICAL GALAXIES WITH DUST LANES
}

\author{
Francesco Bertola \\ Institute of Astronomy \\ University of Padova \\ 35122 Padova \\ Italy
}

\section{INTRODUCTION}

NGC 5128 (Cen A) has been known for many years as a peculiar elliptical, but only recently has it been recognized as the prototype of a new class of object (Bertola and Galletta 1978). This class of galaxy is characterized by an elliptical-like stellar body crossed along the minor axis by a dust lane, and was comprised initially of five objects. The class was extended in a remarkable way by Hawarden et al. (1981), who drew attention also to the existence of ellipticals with the dust lane along the major, and along intermediate axes. Ninety objects are now listed as dust lane ellipticals in the compilation by Ebneter and Balick (1985) and its updated version (Ebneter 1986).

Kotanyi and Ekers (1979) pointed out the tendency of the dust lane to align perpendicularly to the radio source, when present.

Since 1978 it has been realized that the study of this type of object could lead to important information on the intrinsic shape of elliptical galaxies. In fact there is no a priori reason for these galaxies to be oblate spheroids (Binney 1978 ) since in many cases their rotational velocity is too low to generate a flattened system (Bertola 1972, Bertola and Capaccioli 1975, Illingworth 1977).

The dust lane silhouetted against the luminous body of the galaxy, which otherwise would be classified as a pure elliptical, is interpreted as a disk or a ring seen almost edge-on, the result of a past capture by an early type galaxy of a gas rich system. It is therefore clear that the observed configuration depends on the intrinsic shape of the elliptical galaxy and on the impact parameters.

In this paper we shall describe the morphological, statistical, kinematical, and photometric properties, of the elliptical galaxies with dust lanes.

\section{MORPHOLOGY AND DEFINITION OF THE SAMPLE}

The appearence of the dust lane, regardless of its orientation with respect to the stellar body, can be described in the following way:

straight: the dust lane lies exactly along an axis of the stellar body and presum- 
ably represents a disk seen exactly edge-on. A good example is NGC 6702.

curved: a slight curvature is present, suggesting a disk seen almost edge-on. NGC 5485 is an example.

multiple: in a few cases and notably in NGC 1947, a set of parallel dust lanes is present on one side of the galaxy. The lanes could be the result of a system of coplanar rings seen at an angle.

ring-like: a full ring is visible; from its flattening, and on the hypothesis of intrinsic circularity, one can deduce the tilt angle. The best example is NGC 5266 and possible cases are Anon 0151-498 and Anon 0418-583.

warped: the prototype is NGC 5128 where the dust lane, aligned along the minor axis, bends toward the major axis in the outer regions. Additional cases are NGC 5363, NGC 5266, Anon 0151-498, and Anon 1029-459.

In Fig 1 six representative cases are illustrated with high resolution photographs obtained at the prime focus of the $3.9 \mathrm{~m}$ AAT.

A straightforward question arises from the above morphological description: is the dust distributed to form a disk or is it confined to a ring? While in a few cases the ring is clearly visible, in the case of NGC 5128 there is evidence that the gas associated with the dust forms a disk both in ionized (Bland 1985) and neutral (van Gorkom 1986) hydrogen.

Concerning the extent of the dust lane with respect to the galaxy, a variety of situations are encountered. There are galaxies where the dust lane is confined to the innermost regions of the galaxy (e.g. NGC 4374), and galaxies like NGC 5128 where the dust lane can be followed up to the limit of detection of the luminous stellar material. In Table I we have indicated for each galaxy whether the dust extends to the inner, intermediate, and outer regions. One sees that inner and outer dust lanes are present in approximately equal number. Elliptical galaxies with inner dust lanes are easily found with modern detectors and certainly in the future they will outnumber those with extended dust lanes.

Before proceeding to analyze the properties of ellipticals with dust lanes, it is necessary to define the sample according to precise morphological criteria. In order to do this the catalog of dusty elliptical galaxies and its updated version (Ebneter and Balick 1985, Ebneter 1986 ) have been used as basic references, carefully selecting from them the ellipticals with well defined dust lanes. Patchy dust structures were excluded, even those regularly distributed along one axis, as in the case of NGC 1316 (Fornax A).

The first part of Table I lists the elliptical galaxies with dust lanes along the minor axis. Due to the special character of this morphology, the identification of these objects is rather unambiguous. In fact we do not know of any disk galaxy, the alternative to an elliptical, where the bulge is elongated perpendicularly to the plane defined by the disk. However, one should be very careful on this point, since there is a tendency in the usual classification schemes to call S0 any galaxy where a stellar body is crossed by a dust lane, regardless of orientation. Thirty objects are listed in Table I as minor axis dust lane ellipticals. In Ebneter's catalog there are 34 objects listed as minor axis dust lane ellipticals, and 23 of these are in common with those of Table I. Also in Table I there are 6 objects which are classified in different way by Ebneter, while 9 cases given by the latter are discarded.

It is worth mentioning why NGC 3108 has been omitted from Table I; in addition to the dust lane crossing the stellar body along the major axis, there is a trace of a luminous ring associated with the dust. This galaxy is probably the 


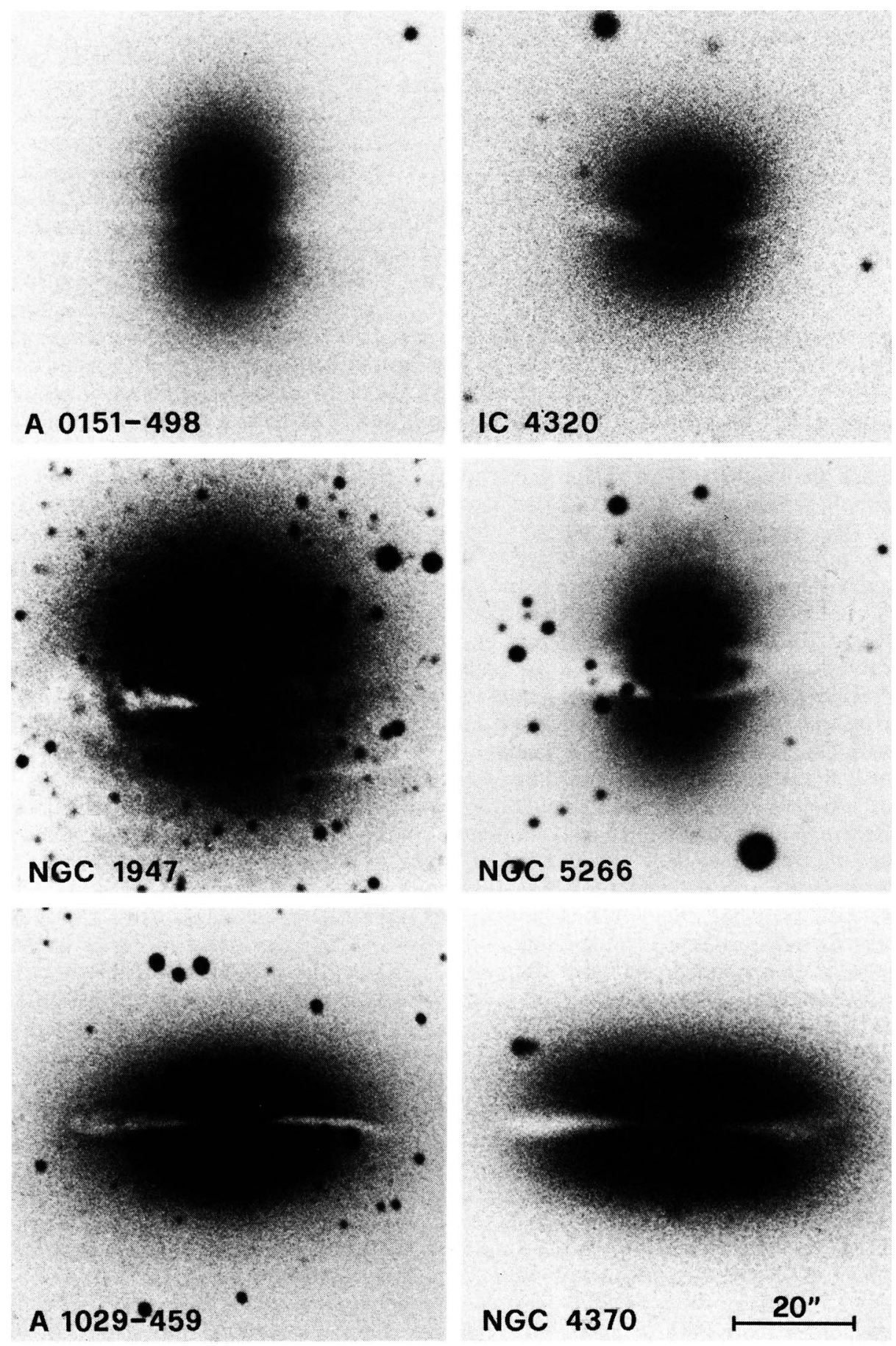

Figure 1. Dust lane ellipticals photographed with the 3.9m AAT. 
Table I. Elliptical galaxies with dust lanes

\begin{tabular}{|c|c|c|c|c|c|c|c|c|c|c|c|}
\hline ident. & Bmag & $V_{r}$ & $M_{B}$ & $b / a$ & $V_{m a x}$ & $\sigma_{0}$ & $V_{\max } / \sigma_{0}$ & $(V / \sigma)_{*}$ & ext & $\Delta \phi$ & Ref. \\
\hline N 185 & 11.00 & -227 & -14.59 & .86 & & & & & $\mathrm{~N}$ & 90 & \\
\hline I 1575 & & & & .75 & & & & & I & 90 & \\
\hline N 404 & 11.30 & -39 & -17.37 & 1.00 & & & & & $\mathbf{N}$ & 90 & \\
\hline N 662 & 13.60 & 5660 & -21.67 & .63 & & & & & $\mathrm{~N}$ & 90 & \\
\hline $0151-498$ & 13.37 & 6170 & -22.09 & .67 & 50 & 257 & 0.19 & 0.27 & $\mathbf{E}$ & 90 & 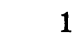 \\
\hline $0206+355$ & 14.69 & 11600 & -20.60 & .87 & & & & & $\mathbf{E}$ & 90 & \\
\hline N 1052 & 11.53 & 1471 & -20.91 & .69 & 96 & 245 & 0.39 & 0.59 & $\mathrm{~N}$ & 90 & 2 \\
\hline N 1297 & 12.61 & 1599 & -19.90 & .91 & & & & & $\mathrm{~N}$ & 90 & \\
\hline $0418-583$ & & & & 1.00 & & & & & $\mathbf{E}$ & 90 & \\
\hline N 1947 & 11.83 & 1160 & -19.88 & .90 & 47 & 150 & 0.38 & 1.12 & $\mathbf{E}$ & 90 & 3 \\
\hline 0609-331 & & 11300 & & .50 & 160 & 265 & 0.60 & 0.61 & $\mathbf{E}$ & 90 & $x$ \\
\hline $0632-629$ & & 8550 & & .57 & 145 & 215 & 0.67 & 0.77 & $\mathrm{E}$ & 90 & 4 \\
\hline $0641-412$ & & 10900 & & .86 & 100 & 160 & 0.63 & 0.41 & $\mathrm{E}$ & 90 & 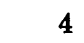 \\
\hline N 2534 & 13.80 & 3517 & -20.44 & .86 & & & & & I & 90 & \\
\hline $1029+544$ & 13.20 & & & .90 & & & & & I & 90 & \\
\hline N 3656 & 13.40 & & & 1.00 & & & & & I & 90 & \\
\hline N 4583 & 14.00 & & & & & & & & I & 90 & \\
\hline N 5128 & 7.96 & 497 & -22.60 & .77 & 40 & 140 & 0.29 & 1.04 & $\mathbf{E}$ & 90 & 5,7 \\
\hline N 5266 & 12.27 & 2880 & -21.92 & .71 & 140 & 200 & 0.70 & 1.09 & $\mathrm{E}$ & 90 & 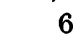 \\
\hline I 4320 & & & & 1.00 & & & & & $\mathbf{E}$ & 90 & \\
\hline $1352-336$ & & & & .80 & & & & & $\mathbf{E}$ & 90 & \\
\hline N 5363 & 11.40 & 1121 & -20.50 & .56 & 135 & 199 & 0.67 & 0.75 & $\mathrm{~N}$ & 90 & \\
\hline N 5485 & 12.40 & 1985 & -20.69 & .74 & & & & & N & 90 & \\
\hline $1459-724$ & & & & .70 & & & & & $\mathbf{E}$ & 90 & \\
\hline N 5898 & 12.60 & 2267 & -20.70 & 1.00 & & & & & $\mathbf{N}$ & 90 & \\
\hline N 6251 & 14.00 & 6900 & -21.70 & .83 & & & & & $\mathbf{N}$ & 90 & \\
\hline N 6702 & 13.80 & 4706 & -21.07 & .79 & & & & & $\mathrm{~N}$ & 90 & \\
\hline $2105-365$ & & & & .54 & & & & & $\mathbf{N}$ & 90 & \\
\hline N 7432 & 15.10 & & & .67 & & & & & I & 90 & \\
\hline N 7625 & 12.80 & 1622 & -20.38 & 1.00 & & & & & I & 90 & \\
\hline N 1199 & 12.42 & 2666 & -21.23 & .60 & & & & & $\mathbf{N}$ & 0 & \\
\hline $0557-524$ & & & & .50 & & & & & I & 0 & \\
\hline $1029-459$ & 1352 & 2760 & -20.10 & .47 & 210 & 260 & 0.81 & 1.27 & $\mathrm{E}$ & 0 & 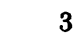 \\
\hline N 3302 & & & & .77 & & & & & $\mathbf{E}$ & 0 & \\
\hline $1040+776$ & & & & .60 & & & & & $\mathrm{E}$ & 0 & \\
\hline N 3665 & 11.60 & 2002 & -21.27 & .86 & & & & & $\mathbf{N}$ & 0 & \\
\hline N 4370 & 14.10 & 468 & -15.76 & .53 & & & & & $\mathbf{E}$ & 0 & \\
\hline N 5745 & & & & .53 & & & & & E & 0 & \\
\hline N 7052 & 14.00 & 46.40 & -20.84 & .56 & & & & & $\mathrm{~N}$ & 0 & \\
\hline $0147-269$ & & & & .57 & & & & & $\mathbf{E}$ & 75 & \\
\hline N 708 & 14.80 & 5047 & -20.22 & .83 & & & & & I & 60 & \\
\hline $0219-345$ & & & & .62 & & & & & $\mathbf{E}$ & 40 & \\
\hline N 4374 & 10.80 & 952 & -21.47 & .80 & 68 & 290 & 0.23 & 0.46 & $\mathrm{~N}$ & 60 & 3 \\
\hline $1307-467$ & & 1853 & & .67 & & & & & I & 30 & \\
\hline N 5799 & & 313 & & 0.09 & & & & & $\mathbf{E}$ & 40 & \\
\hline N 7070A & 13.40 & 2382 & & .40 & $<30$ & 100 & $<0.3$ & $<0.40$ & $\mathbf{E}$ & 35 & \\
\hline
\end{tabular}


Table I: continued The information by column, is as follows:

(1) ... Identification.

2) ... B magnitude from Nilson (1973) and Lauberts (1982).

(3) ... Radial velocity from Palumbo et al. (1983) and from references in 12.

(4) ... Absolute magnitude from B and $V_{r}\left(\mathrm{H}=50 \mathrm{~km} \mathrm{sec}^{-1} \mathrm{Mpc}^{-1}\right)$.

5) ... Axial ratio from Hawarden et al. (1981), Nilson (1973) and Lauberts (1982).

6) ... Rotational velocity.

7) ... Central velocity dispersion.

8) ... Ratio of $V_{\max }$ and central velocity dispersion.

(9) ... Ratio between $V_{\max } / \sigma_{0}$ and the corresponding value for an oblate isotropic rotator.

(10)... Extension of the dust lane with respect to the stellar body. E extended; N confined to nuclear region; I intermediate

(11)... Angle in degrees of the dust lane to the major axis of the galaxy.

(12)... References for kinematical data. For additional references see Ebneter and Balick (1985).

1. Sharples et al 1983; 2. Davies \& Illingworth 1986; 3. Bertola et al. 19864. Möllenhoff \& Marenbach 1986; 5. Bertola et al. 1985; 6. Varnas et al. 1986; 7. Wilkinson et al. 1986.

second instance of an elliptical galaxy with an outer luminous ring, the other being AM 2020-5050, recently discovered by Whitmore at al. (1987).

The second part of the Table I lists nine elliptical galaxies with a dust lane along the major axis. They are all in the catalog by Ebneter (1986), who, however, lists 37 objects belonging to this type. The reason for the drastic reduction lies in the fact that an edge-on S0 galaxy can be easily confused with an elliptical galaxy with a dust lane along the major axis. If the disk-to-bulge ratio in an So galaxy reaches a value such that the luminous disk is at the limit of detection, then it is very difficult to make a distinction. The size of the disk with respect to the bulge plays also a role in the misclassification of major axis dust lane ellipticals. There are cases in which the presence of a disk is missed if the exposure is too low; and vice-versa inner disks in S0 are cancelled out by a deep exposure. Given such difficulties we tried to select only those cases in which the presence of an even tenuous luminous disk can be ruled out. In connection with the relationship between S0 and major axis dust lane ellipticals it is interesting to remark that the morphology of Anon 1029-459 (Fig.1), where the dust lane along the major axis is strongly warped on both sides, closely resembles that of the S0 NGC 5866 (Sandage 1961) with the only difference that the latter possesses an additional luminous disk. Is the origin of the dust lane in these two galaxies the same?

The last part of Table I lists the elliptical galaxies with a dust lane along an axis intermediate between the major and minor axis. Seven cases were selected out of the 18 given by Ebneter (1986). This configuration can be explained by supposing that we are looking at triaxial galaxies at an angle to the planes where the gas and dust are permitted to settle, contrary to what happens in the cases of major and minor axis dust lanes, where the line of sight lies approximately in these planes. An alternative explanation is that we are looking at gas and dust not yet settled.

It is worth mentioning a couple of elliptical galaxies whose morphology is related to that of dust lane ellipticals, viz. NGC 5077 (Bertola et al.1986) and NGC 7097 (Caldwell et al.1986). These are characterized by a narrow distribution 
of ionized gas along the minor and major axis respectively. No dust has been detected. Very probably the same mechanism that has produced the dust lane ellipticals has given rise to such peculiar objects.

NGC 1052, which is listed in Table I as a minor axis dust lane elliptical according to images by Sparks et al. (1986), is characterized by an HI distribution ( van Gorkom et al. 1986 ) much more extended than the dust, but aligned also with the minor axis.

Spindle galaxies with the gaseous ring along the minor axis of the stellar body seem not to be related to minor axis dust lane ellipticals for the following reasons: in several cases the stellar body is clearly not an elliptical galaxy but an S0 with a well defined disk and bulge. In addition there is a tendency for the warps of the gaseous ring (e.g. NGC 4650A) to align with the minor axis of the galaxy in the outer regions, contrary to the behaviour of the minor axis ellipticals. This suggests a different dynamical origin for the two phenomena.

\section{THE DISTRIBUTION OF THE INTRINSIC AXIAL RATIOS}

If elliptical galaxies are triaxial systems, it is possible to derive the distribution $f(b / a, c / a)$ of the intrinsic axial ratios from that of the projected ones, only if an assumption is made on the length of the middle axis (Benacchio and Galletta 1980; Binney and de Vaucouleurs 1981). Considering the sample of major and minor axis dust lane ellipticals it should be possible to put constraints on $f$. In fact, since the permitted planes for the dust are those defined by the major and minor axes each with the intermediate axis, the apparent major axis coincides with the true major axis for the minor axis dust lane ellipticals, and the apparent minor axis coincides with the true minor axis for major axis dust lane ellipticals. In this way the apparent distribution is a function of $f$ and of only one projection angle, which is the angle between the intermediate axis of the galaxy and the line of sight.

The distribution of the intrinsic axial ratios of dust lane ellipticals can be constrained further by considering the relative number of minor to major axis dust lane ellipticals. The number of ellipticals with the dust lane along the minor axis listed in Table $I$ is 30 , while major axis ones are 9, so that the latter galaxies represent $22 \%$ of the total. On the other hand this percentage in the compilation of Ebneter (1986) is $57 \%$, owing to the inclusion among major axis dust lane ellipticals of a large number of galaxies possessing a luminous disk. It should be noted that the survey of Hawarden et al. (1981), which has the advantage of being statistically meaningful since it is based on a survey on the Sky Survey plates, lists 6 major axis dust lane ellipticals (excluding doubtful cases) as against 12 minor axis ones. The fact that in our listing, minor axis dust lane ellipticals outnumber major axis ones leads us to belive that there is an intrinsic paucity of the second ones with respect to the first. As has been mentioned already, the potential of a triaxial galaxy which acquires gas (and dust) from outside will force the gas to orbit in the planes defined either by the minor and intermediate axes, or by the major and intermediate axes. The settling toward one of the two planes depends on the orientation of the initial rotation axis of the gas. The shape of the galaxy defines the fraction of the orientation sphere for which the gas will settle onto one of the two planes ( Steiman-Cameron 1984). It is therefore clear that the total fraction of objects with dust rings or disks perpendicular to the major axis that we observe as minor axis dust lane ellipticals, is a function of the distribution of the intrinsic flattening, 
which is again constrained by an observational quantity. In other words the result on the relative number of minor to major axis dust lane ellipticals would indicate a tendency toward prolateness than toward oblateness for our sample of galaxies. A statistical analysis along these lines is in progress by Bertola,Galletta,Vietri and Zeilinger.

\section{KINEMATICAL AND PHOTOMETRIC PROPERTIES}

There are two different kinematical components in the ellipticals with dust lanes. The first deals with the motions of the stars while the second deals with the gas associated with the dust. As listed in Table I, we possess information on the rotation and velocity dispersion for nine minor axis, one major axis, and two intermediate axis dust lane ellipticals. Data reduction is in progres for NGC 3302, NGC 4370, and NGC 5745 (Bertola et al. 1986).

The ionized gas has been detected in all but two minor axis dust lane ellipticals (Anon 0632-629 and Anon 0641-412). As a general result the rotation axis of the gas is perpendicular to that of the stars. This fact has deep implications on the origin of the gas and dust and gives strong support to the idea that they were acquired from outside. A recent and detailed study (Bland 1985) of the ionized gas in NGC 5128 leads to the conclusion that the mass of the gas is insignificant compared with the mass of the stellar component. At this point it should be mentioned that sixteen ellipticals in our sample were detected by the IRAS satellite. Dust mass estimates (Zeilinger 1986) from these data are in the range of $10^{5} \div 10^{6} M_{\odot}$, so that the mass of the gas could be at least a couple of order of magnitudes larger. In this connection, an analysis on the possible self-gravitation of the dust rings in ellipticals similar to that for polar rings in S0 (Sparke 1986) is desirable.

Unfortunately no emission lines were detected in the only case of a major axis dust lane elliptical that has been studied (Anon 1029-459). If the gas is acquired from outside one would expect to find cases in which the gas is counter-rotating with respect to stars, as in NGC 7097 (Caldwell et al. 1986) and in NGC 4546 (Galletta 1986). From the data in Table I it appears immediately that no slow rotators relative to the isotropic oblate rotator are present among the dust lane ellipticals, although the brightest object reaches $M_{B}=-22.6$. Whether this fact is due to the small sample at our disposal or is an intrinsic property of dust lane ellipticals, is a point to be checked with additional observations, in order to establish whether the nature of dust lane and normal ellipticals is similar or distinct.

It is worth noting that the rotational velocity of the major axis dust lane elliptical Anon 1029-459 is as high as $210 \mathrm{~km} \mathrm{sec}^{-1}$, which is not reached in any other elliptical galaxy. This casts some doubt on whether ellipticals with dust lanes along the major axis, whose numbers have been so drastically reduced in our sample, really exist. This again is a point which needs future observations.

Considerable information on the intrinsic shape of the minor axis dust lane ellipticals can be deduced from kinematical observations. For a minor axis dust lane elliptical the pure oblate configuration is excluded. In fact in this case, if the gas is captured from outside it tends to align with the major axis in the inner regions (Lake and Norman 1983), causing a central discontinuity which is not observed. On the other hand the pure prolate configuration is also excluded in those cases with high values of $V_{\max } / \sigma_{0}$ due to the inability of the models to reproduce the case (Richstone and Potter 1982). Therefore it follows that the shape of a minor axis 
dust lane elliptical is the triaxial one. The rotational velocity which is observed along the major axis of the galaxies is a combination of the streaming velocity of the stars and of the tumbling around an axis in the plane defined by the minor and intermediate axis. In order to give rise to a well defined dust lane, the latter has to see an almost stationary potential. Therefore the conclusion is that the tumbling motion, if any, has to be very small with respect to streaming. Some ideas as to whether we are looking closer to the intermediate axis or to the minor axis, can be derived by the amount of streaming which is higher in the first case than in the second. Then by means of the tensor virial theorem, a lower limit of the axial ratio $b / a$ or $c / a$ can be deduced. If warps are present in the dust lane, an upper limit to these axial ratios can be set by assuming that the warps show the direction of the original infall, on the hypothesis that the warps are transient phenomena (Caldwell 1984; Bertola et al. 1985).

Recent photometric studies of dust lane ellipticals concern NGC 5266 (Caldwell 1984, Varnas et al. 1986) and about twenty other cases whose reduction is in progress (Bertola et al. 1986). All cases are characterized by the fact that the $r^{1 / 4}$ law is more or less followed. It seems that the photometry does not help so much in discriminating S0's from major axis dust lane ellipticals.

\section{THE WARPS AS TRANSIENT OR STATIONARY PHENOMENA}

Tubbs (1980) and later Simonson (1982) have produced a model of NGC 5128 where the dust lane represents material captured from the exterior and the warps are comprised of material not yet settled either in the principal plane of a prolate elliptical, or in the plane defined by the minor and intermediate axis of a triaxial one. In this way the warps are transient phenomena and they are destined to disappear and give rise to an elliptical with a straight dust lane, if tumbling is not present. An alternative interpretation has been given by van Albada, Kotanyi and Schwarzschild (1982), who were able to reproduce the phenomenon as a stationary one by assuming a slow tumbling motion around the minor axis of the triaxial figure of the elliptical body. However, the model requires that the tumbling motion has to be retrograde with the respect to the motions of the warps.

Four minor axis dust lane ellipticals with warps have so far studied, viz. Anon 0151-498, NGC 5128, and NGC 5266 (where the observed motions along the major axis are prograde), and NGC 5363 (with retrograde motion) (Bertola et al. 1985). If tumbling is present, the streaming motions could be either in the same or in opposite way (Freeman 1966, Vietri 1986) so it appears difficult to discriminate between the transient and stationary case. While a satisfactory model has been proposed for NGC 5128 on the first hypothesis, an equally satisfactory alternative model for NGC 5266 assumes counterstreaming with a velocity at $7 \mathrm{kpc}$ twenty times larger than that of tumbling (Varnas et al 1986). As a consequence NGC 5266 would be an almost oblate ( $10 \%$ of triaxiality) galaxy with stable warps. Stability is also suggested by the ordered structure of the dust ring in this galaxy. Of course this does not mean that the gas and dust were not acquired. It is just a matter of how much time has elapsed since the encounter.

There are some other factors that could help in deciding the mechanisms responsible for the warps, as in the case of NGC 5363. In this galaxy the dust is so highly warped in the outer regions that the dust lane runs parallel to the major axis. To explain this in terms of transient warps would require the galaxy to be 
almost prolate. On the other hand the high rotational velocity suggests rather an almost oblate object. This contradiction is not present in the stationary model.

It is a pleasure to thank the Anglo-Australian Observatory for the unique opportunity which has been given to the author in obtaining the high resolution photographs of Fig.1 through their service photography. In preparing the manuscript I benefited discussions with M.Capaccioli, G. Galletta, M.Vietri and W.Zeilinger. Thanks are due to Peter Usher for reading the manuscript.

\section{REFERENCES}

Benacchio, L., and Galletta, G. 1980, M.N.R.A.S., 193, 885

Bertola, F. 1972 Proc. 15th Meeting of It. Astr. Soc., p. 199.

Bertola,F., Bettoni, D., Danziger, J., and Sadler, E. 1986, in preparation.

Bertola, F., and Capaccioli,M. 1975, Ap. J., 200, 439.

Bertola, F., and Galletta,G. 1978, Ap. J. (Letters), 226, L115.

Bertola, F., Galletta, G., and Zeilinger, W.W. 1985, Ap. J. (Letters), 292, L51.

Bertola, F., Galletta, G., and Zeilinger, W.W. 1986, in preparation.

Binney, J.J. 1978, M.N.R.A.S., 183, 501.

Binney, J.J., and de Vaucouleurs, G. 1981 M.N.R.A.S., 194, 679.

Bland, J. 1985, $P h D$ Thesis, University of Sussex.

Caldwell, N. 1984, Ap. J., $278,96$.

Caldwell, N., Kirshner, R.P., and Richstone D.O. 1986 Ap. J., 305, 136.

Davies, R.L., and Illingworth, G.D. 1986, Ap. J., 302, 234.

Ebneter, K. 1986, private communication.

Ebneter, K., and Balick,B. 1985, A. J.,90, 183.

Freeman, K.C. 1966, M.N.R.A.S., 134, 1.

Galletta, G. 1986, The Messenger, in press.

Hawarden, T.G., Elson, R.A.W., Longmore, A.J., Tritton, S.B., and Corwin H.G., Jr 1981, M.N.R.A.S., 196, 747.

Illingworth,G. 1977, Ap. J. (Letters), 218, L43.

Kotanyi, C.G., and Ekers, R.D. 1979, A. and A., 73, L1.

Lake, G., and Norman, C. 1983, Ap. J., $270,51$.

Lauberts, A. 1982, ESO/Uppsala Catalogue, München.

Möllenhoff, C., and Marenbach, G. 1986, A. and A., 154, 219

Nilson, P. 1973, Uppsala General Catalogue of Galaxies, Uppsala.

Palumbo, G.G.C., Tanzella-Nitti, G., and Vettolani, G. 1983 Catalogue of Radial Velocities of Galaxies, New York.

Richstone, D.O., and Potter, M.D. 1982, Nature, 298, 728.

Sandage, A.R. 1961, The Hubble Atlas of Galaxies, Carnegie Inst. of Washington.

Sharples, L.M., Carter, D., Hawarden, T.G., and Longmore, A.J. 1983, M.N.R.A.S., $202,37$.

Simonson, G.F. 1982, PhD Thesis, Yale University.

Sparke, L.S. 1986, M.N.R.A.S., 219, 657.

Sparks, W.B., Wall, J.V., Thorne, D.J., Jorden, P.R., van Breda, I.G., Rudd, P.J., and Jorgensen, H.E. 1985, M.N.R.A.S., 217, 87.

Steiman-Cameron, T.Y. 1984, $\mathrm{PhD}$ Thesis, Indiana University.

Tubbs, A.D. 1980, Ap. J., $241,969$.

van Albada, T.S., Kotanyi, C.G., and Schwarzschild, M. 1982, M.N.R.A.S., 198, 303. 
van Gorkom, J.H. 1986, private communication.

van Gorkom, J.H., Knapp, G.R., Raimond, E., Faber, S.M., and Gallagher, J.S. 1986, A. J., 91, 791.

Varnas, S.R., Bertola, F., Galletta, G., Freeman, K., and Carter, D. 1986, Ap. J., in press.

Vietri, M. 1986, Ap. J., 306, 48.

Whitmore, B., Schweizer, F., and McElroy, D. 1987, This Meeting

Wilkinson, A., Sharples, R.M., Fosbury, R.A.E., and Wallace, .P.T. 1986, M.N.R. A.S., 218, 297.

Zeilinger, W.W. 1986, private communication.

\section{DISCUSSION}

Nieto: You made a distinction between ring and disk. Is it so physically, or does the distinction come from a different optical depth of the dust?

Bertola: Ring-like dust lanes are so called because of their appearance.

Schechter: Do you know of any systematic search for HI in dust lane ellipticals?

Bertola: No. The only cases I know where HI has been detected are NGC 1052 and NGC 5128.

Ebneter: The morphological classifications of dust lanes in my catalog and in the catalog of Hawarden et al. (1981), were based on Sky Survey material. I think it is extremely important for someone to do a deep CCD survey of a large, magnitudelimited sample of $\mathrm{E}$ and "S0" galaxies in order to determine the real distribution of major axis vs. minor axis dust lanes in galaxies which lack a significant stellar disk.

Whitmore: On the question of whether the material is in a disk or in a ring, I would like to comment that in the cases of S0 galaxies with polar rings, and our one case of an elliptical with an outer ring (AM2020-5050; shown in my poster paper, this volume, p. 413), the extent of the gaseous emission is generally much greater than the optical broad band image of the ring. For example, in AM2020-5050, the ring appears to be about $2^{\prime \prime}$ wide in our $V$ image, and yet $H \alpha$ is observed all the way to the center over a range of about $30^{\prime \prime}$. This suggests that while their may be a gaseous disk, star formation may only occur in a narrow region, thus producing a narrow ring.

Kochhar: Why is it that in some cases the dust lanes are confined to the inner parts of the galaxies, whereas in some other cases, the lanes extend to the outer parts?

Bertola: The data I presented are mainly based on a sample of dust lane ellipticals discovered on the Sky Survey prints. They show extended dust lanes as well as inner ones. Recent CCD surveys tend to reveal mainly inner dust lanes. 